\title{
Risk factors for liver rejection: evidence to suggest enhanced allograft tolerance in infancy
}

\author{
M S Murphy, R Harrison, P Davies, J A C Buckels, A D Mayer, S Hubscher, D A Kelly
}

Department of Child Health, The Medical School, University of Birmingham M S Murphy

The Liver Unit, The Children's Hospital, Birmingham

J A C Buckels

A D Mayer

D A Kelly

Department of

Pathology, The

Medical School,

University of

Birmingham

R Harrison

$S$ Hubscher

School of Mathematics and Statistics,

University of

Birmingham

P Davies

Correspondence to: Dr M Stephen Murphy, Institute of Child Health, Nuffield Building, Francis Road, Birmingham B16 8ET.

Accepted 14 August 1996

\begin{abstract}
After liver transplantation, a relatively low intensity immunosuppressive regimen is employed in our unit: after initial triple therapy (prednisolone, azathioprine, cyclosporin), prednisolone is discontinued at three months and azathioprine at one year. A retrospective study was therefore performed to determine the incidence of rejection, and to identify risk factors for rejection in our patient population.

Over a 10 year period, 135 transplants were performed on 109 children. Thirty four $(25 \%)$ were on infants less than 1 year old. Incidences of acute rejection and irreversible chronic rejection were calculated for grafts surviving more than one and four weeks respectively. Acute rejection occurred in 51 of 101 allografts $(50 \%)$, and irreversible chronic rejection in 11 of 91 allografts (12\%). The immunosuppression strategy was not associated with an increased incidence of rejection.

Acute rejection occurred in only eight of 28 allografts $(29 \%)$ in those transplanted during their first year, compared with 43 of $73(59 \%)$ in older children. Logistic and Cox regression analysis supported age at transplantation as a significant risk factor for acute rejection. Irreversible chronic rejection did not occur in any of 24 grafts in patients transplanted before one year, compared with 11 of $67(16 \%)$ in older recipients. This suggests possible enhanced allograft tolerance with transplantation during the first year of life. This unexpected and potentially important finding now requires confirmation in other large patient series, with blind interpretation of post-transplant liver biopsies.

(Arch Dis Child 1996;75:502-506)
\end{abstract}

Keywords: liver transplantation, rejection, tolerance, immunosuppression.

Liver transplantation is an effective treatment for patients with end stage liver disease. One year actuarial survival rates of $80-90 \%$ can now be expected even in infants and young children. ${ }^{1-4}$ This progress has been due to various factors, including improved timing of transplantation, increased experience in medical management, better immunosuppressive regimens, and advances in liver transplant surgery. The use of reduced-size grafts has expanded the population of young patients for whom suitable donors are available, and liver transplantation in the first year of life is now commonplace..$^{3-5}$

Despite these advances, there is still considerable post-transplant morbidity, and retransplantation is often necessary. ${ }^{12}$ Rejection is common and remains an important cause of allograft failure. ${ }^{26}$ Immunosuppression is associated with a high incidence of complications, infection being the most frequent cause of death after transplantation. ${ }^{78}$ We have employed an immunosuppressive regimen which is less intense than those utilised in other centres. ${ }^{1269-12}$ The aims of this study were therefore to determine the impact of rejection on our patient population, and to identify potential risk factors for rejection.

\section{Patients and methods}

PATIENTS

A retrospective study was carried out in June 1993 on the 109 children who had undergone liver transplantation between 1983 and 1993 in the paediatric liver transplant programme in Birmingham. The case records were reviewed, and the histological results were reassessed in cases in which rejection had been reported, in order to systematically grade severity.

Of the 109 children transplanted, 24 required a second transplant, and two a third, so that in total 135 transplants were performed. The recipients ranged in age from 8 weeks to 15 years (median 2.5 years). Reduced size grafts were employed in $74(55 \%)$ of the procedures, and $34(25 \%)$ of the transplants were in subjects less than 1 year old. There were 51 male and 58 female recipients. All donor/recipient matches were $\mathrm{ABO}$ blood group compatible. The median period of posttransplant follow up was 55 weeks (range 0-8.1 years).

The primary disorders leading to transplantation were extrahepatic biliary atresia (51), fulminant hepatic failure (19), cryptogenic cirrhosis (11), $\alpha_{1}$-antitrypsin deficiency (10), tyrosinaemia type 1 (five), Wilson's disease (three), primary oxalosis (two), hepatoblastoma (two), infantile haemangioendothelioma (one), cystic fibrosis (one), Caroli's disease (one), primary sclerosing cholangitis (one), Crigler-Najjar syndrome (one), and propionic acidaemia (one). Indications for retransplantation were graft ischaemia due to vascular occlusion (12), chronic rejection (eight), primary non-function of the graft (four), and graft failure associated with suspected viral hepatitis (two). 
POST-TRANSPLANTATION IMMUNOSUPPRESSION PROTOCOL

After transplantation, initial immunosuppression consisted of triple therapy with corticosteroids, azathioprine, and cyclosporin. Hydrocortisone ( $<10 \mathrm{~kg}$ body weight, $100 \mathrm{mg} ;>10 \mathrm{~kg}$ body weight, $200 \mathrm{mg}$; divided twice daily, administered intravenously) and azathioprine ( $2 \mathrm{mg} / \mathrm{kg}$, once daily, administered intravenously) were started immediately. Cyclosporin (2 $\mathrm{mg} / \mathrm{kg}$, administered intravenously twice daily) was begun within 24 hours, provided that the urine output was satisfactory. When medication was switched to oral, hydrocortisone was replaced with prednisolone $(2 \mathrm{mg} / \mathrm{kg}$ daily). The dose of prednisolone was gradually reduced after two or three weeks, and, except in those patients with active rejection, it was discontinued three months after transplantation. The dose of cyclosporin was adjusted to achieve whole blood trough concentrations in the range $100-300 \mathrm{ng} / \mathrm{ml}$. Concentrations at the upper end of the range were maintained during the early months after transplantation, but those less than $150 \mathrm{ng} / \mathrm{ml}$ were considered adequate after the first year. Azathioprine was discontinued one year after transplantation.

\section{REJECTION: DIAGNOSIS AND TREATMENT}

In the post-transplant period, liver biopsy was performed when indicated on the basis of clinical manifestations and abnormal liver function tests. In practice, most children underwent at least one biopsy in the early weeks. Protocol annual biopsies were also performed.

The diagnosis of acute rejection was based on three main histological features: (1) a mixed portal inflammatory infiltrate; (2) inflammatory damage to bile ducts; (3) venous endothelial inflammation. ${ }^{1314}$ Each of these three features was graded semiquantitatively on a scale of 0 to 3 ( $0=$ absent; $1=$ mild; $2=$ moderate; $3=$ severe). The individual scores were added together to produce a final rejection score of $0-9$. This score was converted to a rejection grade as follows: $0-2=a b s e n t ; 3=$ borderline; $4-5=$ mild; $6-7=$ moderate; $8-9=$ severe..$^{15}$ The diagnosis of chronic rejection was based on the demonstration of interlobular bile duct loss from at least $50 \%$ of portal tracts. For the purposes of this study we examined the incidence of irreversible chronic rejectionthat is, chronic rejection that eventually led to graft failure-in order to avoid difficulties of histological interpretation in borderline cases of chronic rejection.

Based on the degree of clinical and biochemical disturbances present and on the severity of the histological changes, acute rejection was treated with intensified immunosuppression. Treatment consisted of a three day course of methylprednisolone (20-40 $\mathrm{mg} / \mathrm{kg}$ once daily, intravenous). One patient with refractory rejection was treated with OKT3. Seven patients who showed signs of steroid resistant rejection were given FK506 instead of cyclosporin.
VIRAL SURVEILLANCE

Donor and recipient cytomegalovirus (CMV) status and recipient Epstein-Barr virus (EBV) status were determined serologically before transplantation, and at regular intervals thereafter. Diagnosis of infection was based on the demonstration of significant changes in serological titres. In some cases further evidence of infection was available based on viral culture or immunohistochemistry.

\section{STATISTICAL ANALYSIS}

The following parameters were evaluated as possible risk factors for the development of rejection: the age and sex of recipient and donor, the type of graft employed (whole $v$ reduced size), the occurrence of CMV or EBV infection, the recipient's nutritional status (height for age, weight for age, skinfold thickness), the primary indication for transplantation, and transplantation date. In order to minimise possible confounding effects of early graft loss from other causes, the incidences of acute rejection and irreversible chronic rejection were analysed in those grafts that survived for more than one week or four weeks respectively. Donor and recipient age and sex, graft type, and transplantation date were investigated as potential risk factors for acute rejection by logistic regression for the probability of rejection, and Cox regression for the survival time in days from transplantation to the onset of rejection as implemented in the EGRET statistical package (Statistics and Epidemiology Research Corporation, Seattle, WA, USA).

\section{Results}

Of the 135 allografts employed in these patients, 34 were lost during the first week and 44 during the first four weeks after transplantation. These grafts failed because of primary non-function, ischaemic injury, or patient death.

Acute rejection occurred in $51(50 \%)$ of the 101 allografts which survived beyond the first week, and a total of 60 separate episodes of acute rejection were documented. The changes were graded as mild in 20 , moderate in 25 , and severe in six cases. The median interval from transplantation to the diagnosis of acute rejection was nine (range 3-44) days. In 48 of the 51 affected grafts (94\%) the process was first identified within 21 days of transplantation, at which time all were still receiving high dose prednisolone (1.5-2 $\mathrm{mg} / \mathrm{kg}$ per day). In 42 cases there was just one episode of acute rejection, but in nine there was a second episode, occurring between two and 54 (median nine) weeks after the first. Acute rejection occurred in four cases after discontinuation of corticosteroids at three months, but in two of these the doses of azathioprine and cyclosporin had also been reduced because of suspected infection.

In total, 40 of the 51 allografts $(78 \%)$ with histological acute rejection required courses of treatment with methylprednisolone (intravenous injection). In 30 a single course was necessary, seven required a second, and three a third. Methylprednisolone treatment 


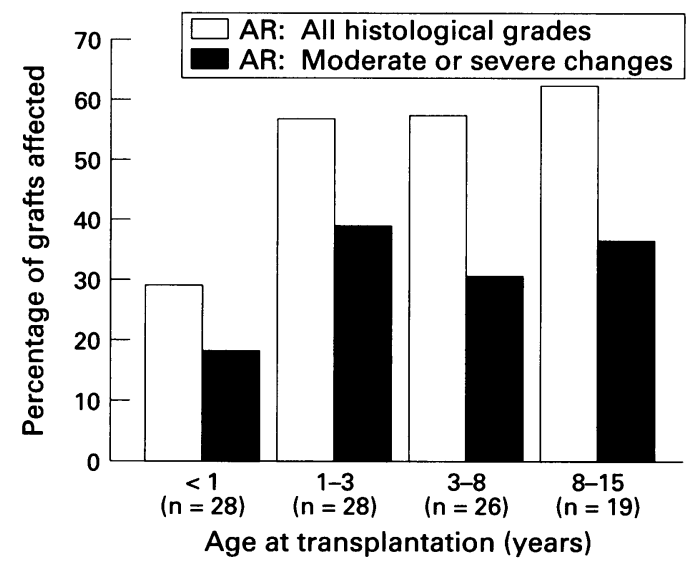

Figure 1 Incidence of acute rejection $(A R)$ in different transplant recipient age groups.

achieved resolution of acute rejection in 31 cases $(78 \%)$. Three of seven steroid refractory cases responded to FK506 rescue treatment. One patient received a course of OKT3 monoclonal antibody treatment without resolution of rejection. Of those allografts with documented acute rejection, $22 \%$ later developed irreversible chronic rejection.

Irreversible chronic rejection occurred in 11 $(12 \%)$ of the 91 allografts that survived beyond four weeks. Acute rejection had previously been documented in all of these cases, and in nine there appeared to have been a gradual evolution from acute rejection to irreversible chronic rejection. The median interval from transplantation to the diagnosis of irreversible chronic rejection was 14 (range 5-30) weeks. One case occurred in a child retransplanted for previous irreversible chronic rejection. Of the 11 cases of irreversible chronic rejection, eight led to retransplantation, and three resulted in patient death.

\section{IMMUNOSUPPRESSION ASSOCIATED} COMPLICATIONS

There was no significant increase in the incidence of infection in those patients treated for acute rejection. Of those who required intravenous injection of methylprednisolone, $54 \%$ were treated intravenously with antibiotics for suspected or proved bacterial sepsis in the post-transplant period, compared with $58 \%$ of those who did not. Similarly there was no difference in the incidence of CMV or EBV infection in those given methylprednisolone. The incidence of CMV infection was $36 \%$ in both groups, with donor seropositivity having been closely comparable- $37.5 \%$ in those who required methylprednisolone, and $40 \%$ in those who did not. EBV infection occurred in $42 \%$ of those who received methylprednisolone, compared with $46 \%$ in those who did not. Clinically overt herpes simplex infection occurred in three children, none of whom had received methylprednisolone. Finally, of those who required methylprednisolone, only $17 \%$ were treated with amphotericin for suspected or proved fungal infection, compared with $40 \%$ of those who did not. No cases of posttransplantation lymphoproliferative disease occurred.

\section{RISK FACTORS FOR REJECTION}

Figure 1 shows the proportion of allografts affected by acute rejection in those transplanted during the first year of life compared with older recipient age categories. Acute rejection occurred in only eight of 28 allografts $(29 \%)$ in patients transplanted during the first year, compared with 43 of $73(59 \%)$ in older recipients $\left(p<0.007\right.$ by $\chi^{2}$ test). This trend towards a reduced incidence of acute rejection in those under 1 year was still evident if cases of mild acute rejection were excluded from the analysis, moderate to severe rejection occurring in five of 28 allografts $(18 \%)$ in those less than 1 year and 26 of $73(36 \%)$ in the older recipients ( $p=0.08$ by $\chi^{2}$ test). As stated previously, seven children with steroid resistant acute rejection were treated with FK506, and three of these responded. One of these three was less than 1 year of age, and the others were 13 and 14 years old. Irreversible chronic rejection did not occur in any of 24 allografts in patients transplanted before 1 year, compared with 11 of $67(16 \%)$ in older recipients $\left(p<0.04\right.$ by $\chi^{2}$ test).

On investigation by logistic regression for the probability of rejection and Cox regression for the survival time in days from transplantation to the onset of rejection, patient age at transplantation emerged as a significant risk factor for acute rejection. Figure 2 shows survival curves representing the time interval to the development of acute rejection for three separate recipient age groups (0-1 year, 1-3 years, over 3 years) during the first 45 days after transplantation. The numbers of subjects in these groups were 28,28 , and 45 respectively; and the percentages developing acute rejection were 29,57 , and 60 . The median numbers of days to rejection in these subject groups were 12 (95\% confidence interval (CI) 7 to 35$), 10$ (95\% CI 7 to 14$)$, and 8 (95\% CI 7 to 9 ). The reduced risk for acute rejection in the younger patients is confirmed by the statistical significance of the difference in survival curves $(\mathrm{p}<0.03)$.

There was no significant correlation between indicators of nutritional status, including weight, height, and subscapular and triceps skinfold measurements, before transplantation and the subsequent incidence of acute rejection or irreversible chronic rejection. Height $\mathbf{Z}$ scores at transplantation were lower in those who did not develop acute rejection $(-1.6 v$ $-0.5)$, but this difference was not significant. There was no apparent association between either CMV or EBV infection and the development of acute rejection or irreversible chronic rejection. Neither were any of the various primary liver disorders associated with an increased risk of rejection after transplantation.

\section{Discussion}

Rejection is an important cause of morbidity and mortality after liver transplantation. Acute rejection is treated with intensified immunosuppression, but unfortunately this may cause significant complications. Chronic rejection often leads to allograft failure and in these cases retransplantation is necessary if the patient is to 


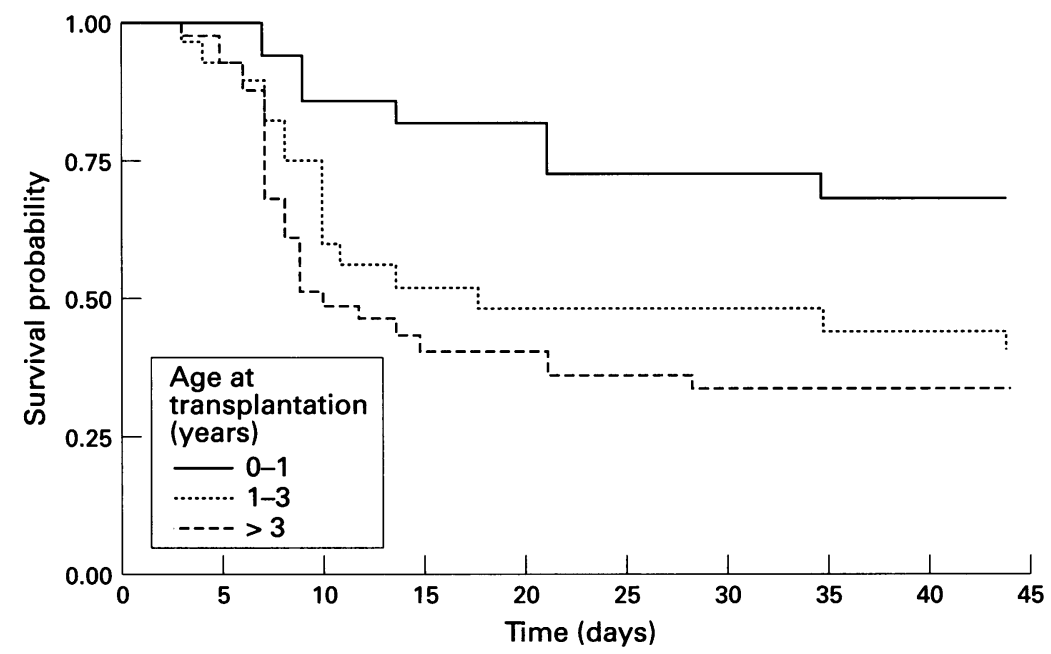

Figure 2 Survival curves representing time to the development of acute rejection during the first 45 days after transplantation. Patients are grouped by age at transplantation: $0-1$ year $(n=28) ; 1-3$ years $(n=28) ;>3$ years $(n=45)$. The role of age as a risk factor for acute rejection is supported by the statistical significance of the difference between the survival curves $(p<0.03)$. later developed chronic rejection. ${ }^{6}$ In our series no such difference was seen, the median interval to the development of acute rejection being seven (range 3-44) days in those who developed irreversible chronic rejection and nine (range 3-35) days in those who did not. Irreversible chronic rejection occurred in $12 \%$ of allografts in our series, compared with reported incidences of approximately $5-10 \%$ in other paediatric liver transplant series, although the exact definition of chronic rejection undoubtedly varied. ${ }^{126}$

In our patients acute rejection began in $94 \%$ of cases within 21 days of transplantation while patients were still receiving corticosteroid treatment. This pattern is similar to that reported in other series. ${ }^{12122}$ Only four of a total of 60 documented episodes of acute rejection in our patients occurred after discontinuation of prednisolone, and in two of these cases the patients were on reduced doses of azathioprine and cyclosporin because of suspected infections. Importantly, in nine of the 11 cases of irreversible chronic rejection in our series, persistent acute rejection had already begun in the early weeks after transplantation, and so prednisolone had not been discontinued in these patients. The practice of withdrawing steroids at three months did not therefore appear to contribute significantly to the incidence of acute rejection or irreversible chronic rejection in our patients.

Although several studies have addressed the subject of risk factors for liver rejection, these have mainly focused on adult patients. This is the first study specifically designed to identify risk factors for rejection in a large group of infants and children undergoing transplantation. In previous studies, suggested risk factors for chronic rejection have included CMV infection, omission of azathioprine from the immunosuppressive regimen, and retransplantation for chronic rejection. ${ }^{23-27}$ In a study of adults in the Birmingham programme, chronic rejection recurred in $27 \%$ of patients retransplanted because of chronic rejection. ${ }^{20}$ Others have reported recurrence rates as high as $90 \% .{ }^{27}$ In contrast, in our series only one of eight children transplanted for irreversible chronic rejection had a recurrence of this disorder in the second graft. In our study the incidences of CMV and EBV infection were not significantly higher in those who developed either acute or irreversible chronic rejection.

The most surprising observation in this study was that transplantation during the first year of life was associated with a significantly reduced risk of rejection. Among those transplanted before 1 year of age, the incidence of acute rejection was approximately half of that in older recipients, and graft failure from chronic rejection did not occur. The published literature has not previously suggested that allograft rejection is less common after liver, cardiac, or renal transplantation in infancy. ${ }^{310} 28-30$ Previous reports of experience in paediatric liver transplantation clearly document the occurrence of rejection even in very young recipients, but these series have generally included relatively few infants, and age has and in nine gradual progression from acute irreversible chronic rejection was observed. Freese et al reported an earlier onset of acute rejection after transplantation in those who 
not been examined as a risk factor. ${ }^{31028}$ Our finding was therefore an unexpected one.

If age at transplantation is truly a significant risk factor for rejection, this would be of great biological interest and an important clinical observation. However, given that this is a retrospective study covering a period of 10 years, caution is necessary in interpreting the results. With the introduction of reduced size liver grafts, infant transplantation gradually became more frequent during the period covered by this study. If, in parallel with this change in transplantation age, there were to have been unrecognised but significant changes in clinical practice or subtle changes in the histological criteria employed in the diagnosis of rejection, this might have led to a non-causal association between age at transplantation and rejection risk. For this reason, the statistical analysis performed in this study was designed to take account of any unrecognised effects that might be linked to transplantation date. Age at transplantation emerged as an independent risk factor for rejection. Nevertheless, in order to confirm or refute the validity of this observation, it is essential that studies should now be performed on other large paediatric transplant populations, in which all post-transplant biopsies are assessed at the end of the study by an individual histopathologist, blinded to patient age.

If age is confirmed as a risk factor for rejection, the biological basis for this phenomenon would be of great interest. A recent study of adult patients in the Birmingham programme demonstrated a significantly lower incidence of chronic rejection in recipients over 30 years of age. ${ }^{23}$ It is possible that this might have been due to an age related alteration in immune function, with reduced alloreactive responsiveness in older patients. ${ }^{31}$ The phenomenon whereby allograft tolerance is readily induced in laboratory animals in early life may be relevant to our findings. ${ }^{32}$

1 Ascher NL, Stock PG, Bumgardner GI, Payne WD, Najarian JS. Infection and rejection of primary hepatic transplants in 93 consecutive patients treated with triple transplants in 93 consecutive patients treated with triple
immunosuppressive therapy. Surg Gynecol Obstet immunosuppressive

2 Busuttil RW, Seu P, Millis JM, et al. Liver transplantation in children. Ann Surg 1991;213:48-57.

3 Esquival CO, Nakazato P, Cox K, Concepcion W, Berquist W, Russell TR. The impact of liver reductions in paediatric liver transplantation. Arch Surg 1991;126:1278-86.

4 Beath SV, Brook GD, Kelly DA, et al. Successful liver transplantation in babies under 1 year. $B M \mathcal{F} 1993 ; 307: 825-8$.

5 Langas AN, Wagner CM, Inagaki M, Stratta RJ, Wood RP, Shaw BW. The results of reduced-size liver transplantation, including split livers, in patients with end-stage liver disease. Transplantation 1992;53:387-91.

6 Freese DK, Snover DC, Sharp HL, Gross CR, Savick SK Payne WD. Chronic rejection after liver transplantation: study of clinical, histopathological, and immunological features. Hepatology 1991;13:882-91.
7 Colonna JO, Winston DJ, Brill JE, et al. Infectious complications in liver transplantation. Arch Surg 1988;123: 360-4.

8 Mor ET, Solomon H, Gibbs JF, et al. Acute cellular rejection following liver transplantation: clinical pathologic feature and effect on outcome. Semin Liver Dis 1992;12:28-40.

9 Lake JR, Roberts JP, Asher NL. Maintenance immunosuppression after liver transplantation. Semin Liver Dis 1992;12:73-9.

10 Sokal EM, Veyckemans F, de Ville de Goyet J, et al. Liver transplantation in children less than 1 year of age. $f$ Pediatr 1990;117:205-10.

11 Shaw BW, Wood RP, Kaufman SS, et al. Liver transplantation for children: part 2. F Pediatr Gastroenterol Nutr 1988; 7:797-815

12 Salt A, Noble-Jamieson G, Barnes ND, et al. Liver transplantation in 100 children: Cambridge and King's College Hospital series. BMF 1992;304:416-21.

13 Snover DC, Sibley RK, Freese DK, et al. Orthotopic liver transplantation: a pathological study of 63 serial liver biopsies from 17 patients with special reference to the diagnossies from 17 patients with special reference to the diagnos-
tic features and natural history of rejection. Hepatology tic features and

14 Hubscher SG, Clements D, Elias E, McMaster P. Biopsy findings in cases of rejection of liver allograft. $\mathcal{F}$ Clin Pathol 1985;38: 1366-73.

15 Hubscher SG, Buckels JAC, Elias E, McMaster P, Neuberger J. Vanishing bile-duct syndrome following liver transplantation-is it reversible? Transplantation 1991;51: 1004-10.

16 Hubscher SG. Pathology of liver allograft rejection. Transplant Immunol 1994;2:118-23.

17 Kusne S, Dummer JS, Singh N, et al. Infections after liver transplantation. An analysis of 101 consecutive cases. Medicine (Baltimore) 1988;67:132-43.

18 Boon AP, Adams DH, Buckels J, McMaster P. Cerebral aspergillosis in liver transplantation. $\mathcal{F}$ Clin Pathol 1990;43: 114-8.

19 Mora NP, Cofer JB, Solomon H, et al. Analysis of severe infections (INF) after 180 liver transplants: the impact of amphoteracin B prophylaxis for reducing the incidence and amphoteracin B prophylaxis for reducing the incidence and 30 .

20 Adams DH, Neuberger JM. Patterns of liver allograft rejection. $\mathcal{F}$ Hepatol 1990;10:13-9.

21 Edmond JC, Thistlethwaite JR, Baker AL, et al. Rejection in liver allograft recipients: clinical characterization and management. Clin Transplant 1987;1:143-50.

22 Klintmalm GB, Nery JR, Husberg BS, Gonwa TA, Tillery GW. Rejection in liver transplantation. Hepatology 1989;10: 978-85.

23 Candinas D, Gunson BK, Nightingale $P$, Hubscher $S$ McMaster P, Neuberger JM. Sex mismatch as a risk factor for chronic rejection of liver allografts. Lancet 1995;346: for chron

24 Manez R, White LT, Linden P, et al. The influence of HLA matching on cytomegalovirus hepatitis and chronic rejection after liver transplantation. Transplantation 1993;55 1067-71.

25 van Hoeck B, Wiesner RH, Ludwig J, Gores GJ, Moore SB, Krom R. Combination immunosuppression with azathioprine reduces the incidence of ductopenic rejection and vanishing bile duct syndrome after liver transplantation. vanishing bile duct syndrome

26 O'Grady J, Alexander GJ, Sutherland S, et al. Cytomegalovirus infection and donor/recipient HLA antigens: interdependent co-factors in pathogenesis of vanishing bile duct syndrome after liver transplantation. Lancet 1988;ii:302-5.

27 van Hoeck B, Wiesner RH, Krom RAF, Ludwig J, Moore SB. Severe ductopenic rejection following liver transplantation: incidence, time of onset, risk factors, treatment, outcome. Semin Liver Dis 1992;12:41-50.

28 Najarian JS, Frey DJ, Matas AJ, et al. Renal transplantation in infants. Ann Surg 1990;212:353-65.

29 Starnes VA, Bernstein D, Oyer PE, et al. Heart transplantation in children. $\mathcal{F}$ Heart Transplant 1989;8:20-6.

30 Esquivel CO, Koneru B, Karrer F, et al. Liver transplantation before 1 year of age. $\mathcal{F}$ Pediatr 1987;110:545-8

31 Lesourd BM, Meaume S. Cell mediated immunity changes in ageing, relative importance of cell subpopulation switches and of nutritional factors. Immunol Lett 1994;40 235-42.

32 Billingham RE, Brent L, Medawar PB. 'Actively acquired tolerance' of foreign cells. Nature 1953;172:603-6. 